\title{
CARElink: Emergency Medical Information Management System
}

\author{
Ingrid Rodríguez Hernández ${ }^{1}$, Jorge L. Cruz Agosto ${ }^{2}$, and Idalides Vergara-Laurens ${ }^{3}, \mathrm{PhD}$ \\ Department of Electrical and Computer Engineering, Universidad Ana G. Méndez, Recinto de Gurabo, Puerto Rico \\ 1irodriguez335@email.suagm.edu, ${ }^{2}$ jcruz654@email.suagm.edu, ${ }^{3}$ ivergara@ suagm.edu
}

\begin{abstract}
Millions of calls related to medical emergencies are placed in 9-1-1 system in the United States every year. The response time are critical in these cases, since a slight delay could be life threatening. Thus, faster response time is important. This work presents a system that allows a paramedic to access a patient's medical history in real-time. This system will securely store a person's medical history in a wearable device. When the paramedics intervene with the patient during an emergency, they will use a mobile device to scan and access the patient's device. Then, the paramedics will have access to the patient's medical information. Future work will include hardware and software testing, performance validation and security strategies.

Keywords-Emergency Medical Services, Prehospital Emergency Care, Medical Information Management, Paramedic Assistance, Medical Portable Device.
\end{abstract}

\section{INTRODUCTION}

According to NENA, the 9-1-1 Association, more than 240 million 9-1-1 calls are placed every year in the United States [1]; approximately, 5\% of those are composed by critical medical incidents such as cardiac and neurological emergencies [2]. The early stage in response time interval is especially critical, as the evidence from the literature concludes that for every minute delay in primary response for certain lifethreatening medical emergencies, there is a measurable effect on mortality [2][3]. According to Blanchard et al. [3], the first 5 minutes in the response interval, where rapid intervention makes the greatest difference. One-minute delay in response time increases the mortality rate by 1 to $2 \%$ and increases hospital treatment costs by $7 \%$ [2]. A solution that could reduce response times by only one minute on average would save thousands of lives per year across the nation. Therefore, faster response times does matter.

One significant challenge to be faced in an emergency, that affects mortality, is the limitation that the paramedics have while handling the patient's medical history. This information is provided by the person who needs the assistance. However, the question is: what happens if the patient is not able to speak, is deaf or unconscious? These situations may affect the emergency response time, compromising a person's life [2]. Furthermore, the risk for improper handling will increase, not only for a caused delay due to limited communication, but also for missing or inaccurate information.

Digital Object Identifier (DOI):

http://dx.doi.org/10.18687/LACCEI2019.1.1.268

ISBN: 978-0-9993443-6-1 ISSN: 2414-6390
This work presents a system to improve the response time to preserve a life. This system assists paramedics during medical emergencies. The proposed system will securely store a person's medical history in a wearable device. The device information is linked with the patient's account. When the paramedics intervene with the patient during an emergency, they will use a mobile device to scan the patient's device. The application will load the patient's information to the paramedic's device. The following sections present the background, the system architecture, the software implementation, and the project's summary.

\section{BACKGROUND}

\section{A. Research and statistics}

Ideally, emergency response times would be minimal for every 9-1-1 call. However, certain emergency calls are known to be more time-sensitive than others, and every second counts [4]. According to Blackwell and Kaufman [6], Pons et al. [5], and Blanchard et al. [3], small variations in time savings affecting a response greater than the 8-minute EMS guideline do not have a substantial effect on patient outcomes. They agree that time reductions affecting the initial response time window have the greatest influence on mortality. These studies focus primarily on response times surrounding an 8-minute guideline. However, all three of these authors concurred that the first 4 to 5 minutes following the incident have a significant impact on mortality. Their studies conclude that there is a positive interrelation between response times and mortality rates.

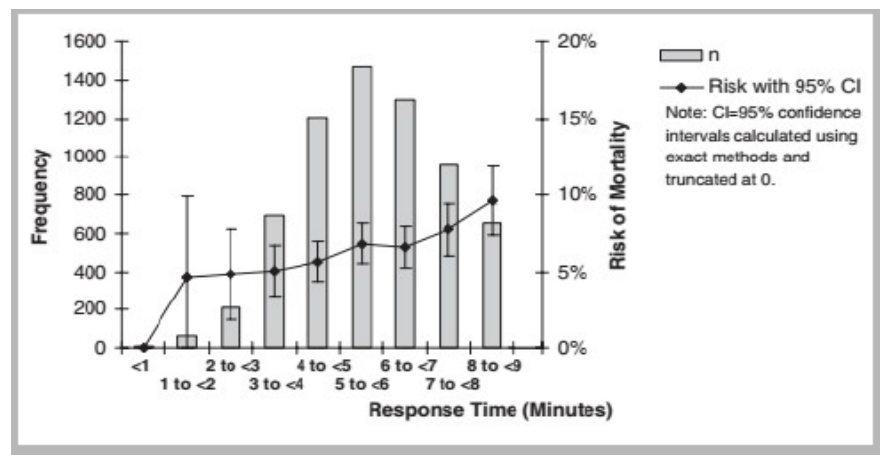

Fig. 1 "Crude risk of mortality over response time, in minutes. $\mathrm{CI}=$ confidence interval" [3]

Blanchard data illustrates a positive trend between increased response times and risk of mortality during the early time window. He found a $1.9 \%$ difference in the risk of mortality for response times $>=4$-minutes ${ }^{[3]}$ (See Fig. 1).

17th LACCEI International Multi-Conference for Engineering, Education, and Technology: "Industry, Innovation, And Infrastructure for Sustainable Cities and Communities", 24-26 July 2019, Jamaica. 


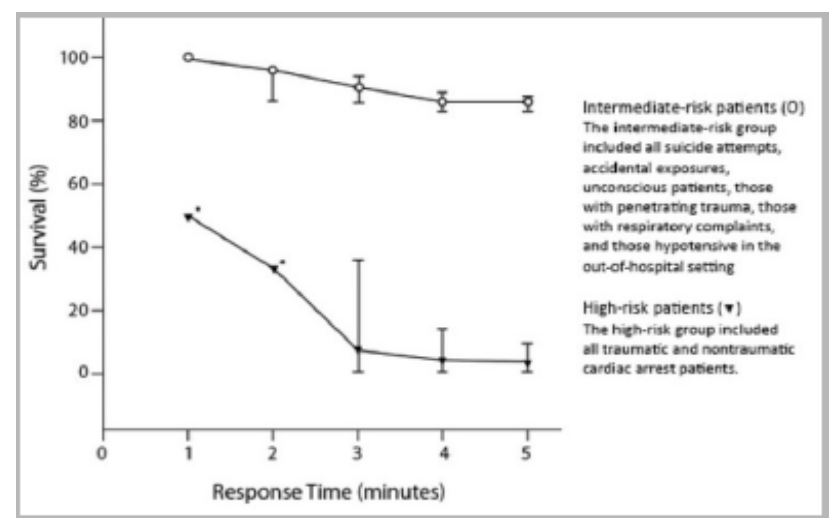

Fig. 2 "Percentages of survival to hospital discharge over paramedic response time and stratified by response groups (bars represent 95\% CIs)" [5]

Pons calculated an odds ratio for mortality of 0.70 when response times were less than four minutes [5]. According to Fig. 2, the drop on survival rate depends on the type of medical emergency. In addition, Fig. 3 shows that the longer response time during the initial 5 minutes, the higher mortality probability.

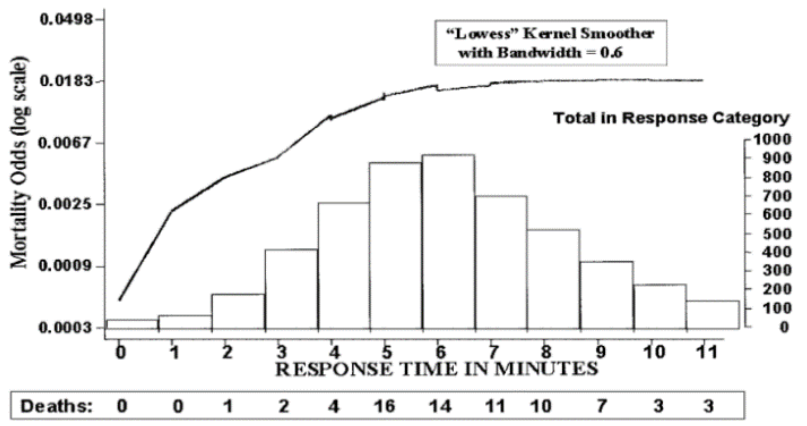

Fig. 3 "Smoother mortality odds by emergency medical services (EMS) response time (Call to Scene)" [6]

\section{SYSTEM ARCHITECTURE}

CARElink is a system that improves the response-time during a medical emergency. The main components are presented in figure 4 . The system is composed by a wearable device to store patient's medical information (e.g., food allergies, medicine allergies, medical conditions), a patients' mobile application to update medical information, a paramedics' mobile application to access the patient's medical information during an emergency.

The following procedure illustrates the system's functionality:

1) The patient acquires the wearable device and downloads the application to register account.

2) Once the account is registered, the patients will be able to fill out the necessary information, such as: demographic information, emergency contact, medical conditions, current medications, and allergies.

3) The information entered will be uploaded and stored in the patient's device, since the account will be linked with the portable device through the application. At this point, the information will be available for an emergency. Also, the information could be uploaded at any time. The account information will be stored in a database as back-up.

4) In case of an emergency, when the paramedics arrive to the scene, they will open an encounter and will scan the patient's device. The information stored in the portable device will be automatically uploaded in the corresponding slots of the opened encounter on the paramedic's tablet.

5) After the paramedics finish their evaluation, the summary report will be submitted and stored in a global database and in the patient's account.

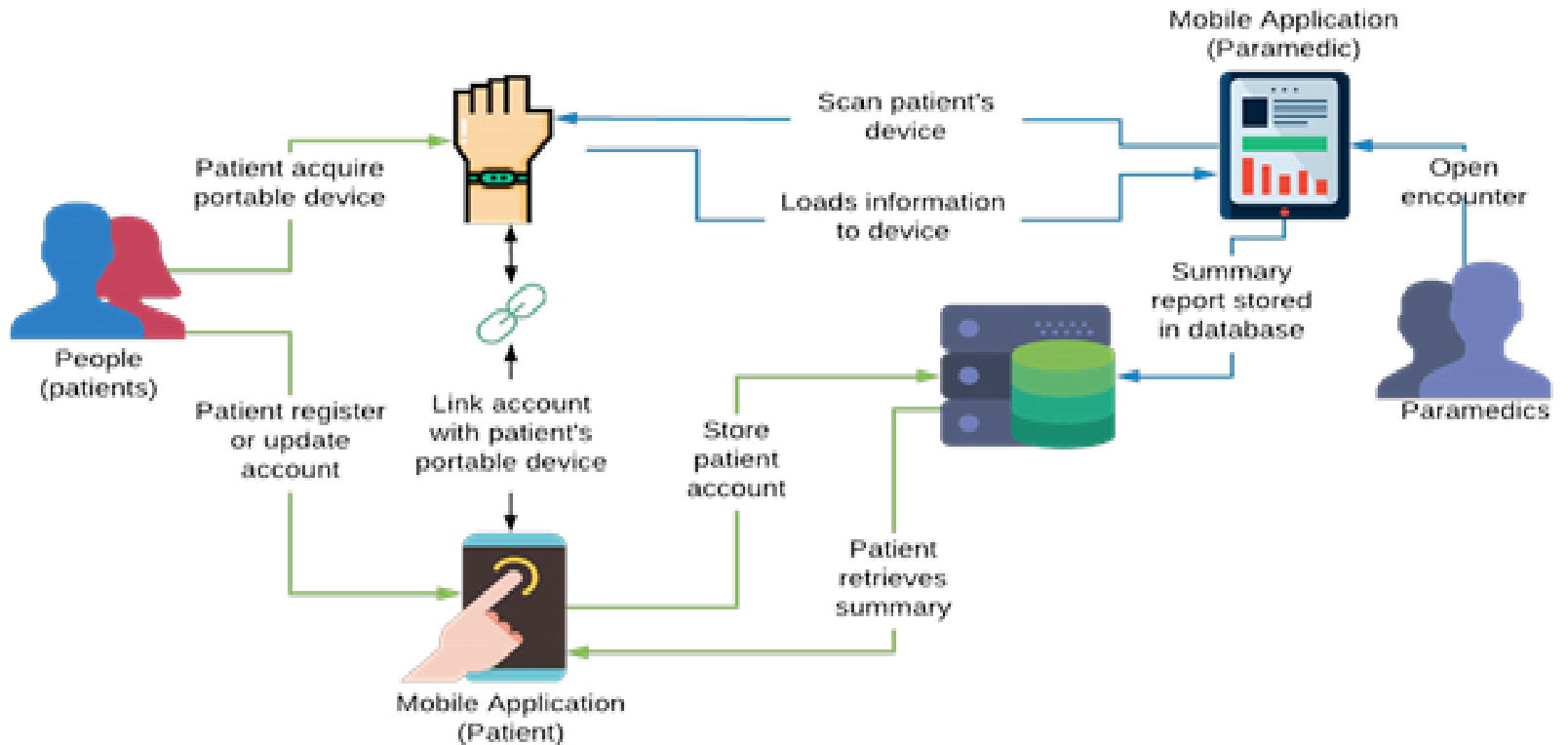

Fig. 4 Main Concept Diagram

\section{A. Hardware}

17th LACCEI International Multi-Conference for Engineering, Education, and Technology: "Industry, Innovation, And 
The wearable device must be compact, not depend on telecommunications, presents a low-energy consumption, cost effective, and data must be accessible when required. Figure 5 shows the preliminary design for the wearable device.

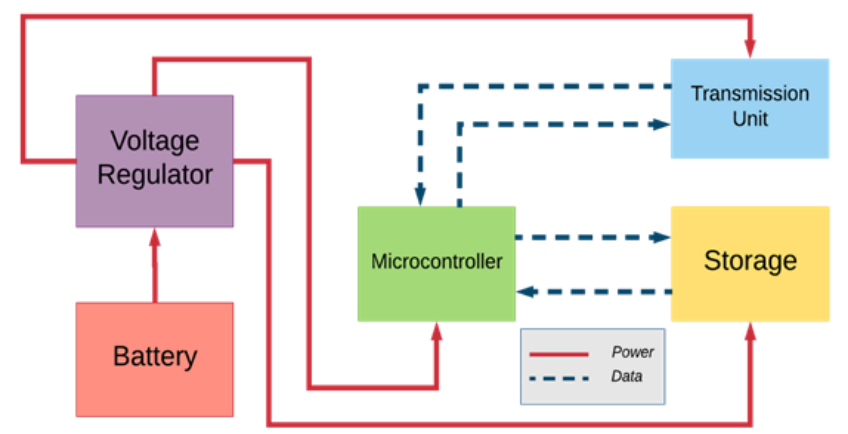

Fig. 5. Initial design

However, as the application was developed, we realized that USB technology is suitable for CARElink because it is compact, does not depend on telecommunications, is cost effective, information remains accessible and does not consume power while it is switched off, and it does not require a battery.

There worth in notice that since time management is so critical during any emergency, the wearable device included an RFID as shown in figure 6 . This will allow the paramedic to track the portable device and verify if the patient has one without wasting valuable time. If the patient has the wearable device, the paramedics will connect their tablet to the device in order to access patient's medical information.

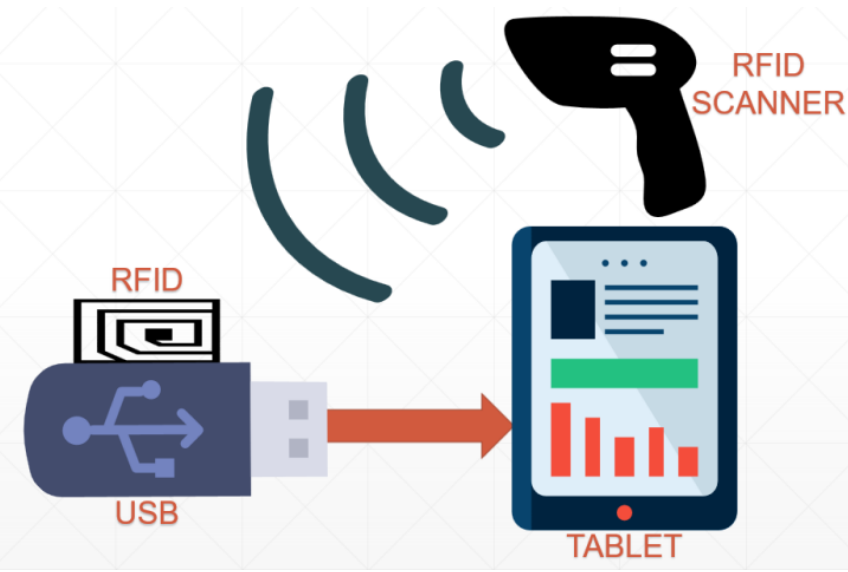

Fig. 6. Hardware components.

\section{B. Software architecture}

CARElink defines three major software components: a patients' mobile application to record medical information, a paramedic's mobile application to access patient's medical information, and an administrative application. In addition, the system will include a global database that not only will store the patient's medical history and demographics, but also it will store the emergency encounter's information and evaluation.

The wearable device will need a smaller local database to store and update the patient's information. In order to avoid synchronization issues when transferring data from the wearable device to the paramedic's device, the entity-relation architecture from the portable device database is designed as a subset of the global database.

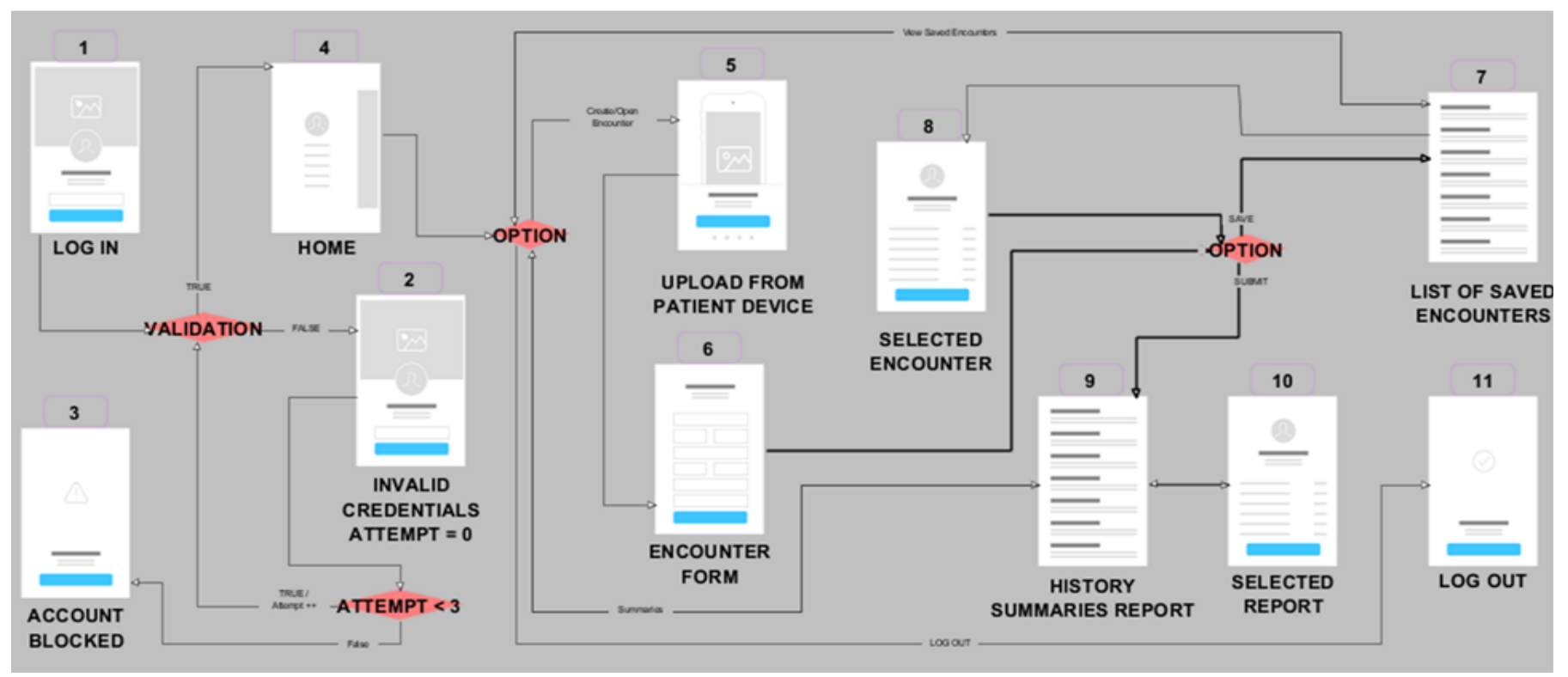

Fig 7. Patient's application workflow 


\section{SOFTWARE IMPLEMENTATION}

This section presents the patients' applications and the paramedics' application.

\section{A. Patients' application}

Figure. 7 presents the patients' application workflow. Each step works as follows:

1) Login Screen: there are three options: Registration, Sign In, and Recover account.

2) Terms and Conditions: To register a new account, press link on the login screen and the user will be directed to the Terms and Conditions page. Once the user agrees to it, it will direct the patient to the next step.

3) Pair device with account: For a user being able to register it will need to prove that has a legitimate portable device. At this point, the user's portable device must be linked to account.

4) Unsuccessful registration: This page will appear if the device is not successfully linked. If this page appears it will redirect the user to the Login Screen (1).

5) Registration Form: In this page, the user will fill out the necessary information to register account.

6) Confirmation Information: this page will show all the information the user entered in the previous page Registration Form (5). If the information is not correct, the user will be able to go back and edit information. Otherwise it will submit it. Once done it will be redirected to the Login Screen (1).
7) Invalid Password: If the username and password validation is unsuccessful in Login Screen (1) it will go to this page. The user will have 3 try attempts. After three unsuccessful attempts, the user will be allowed to retrieve the account in the Recover Password Screen (8). Otherwise, it will direct the user to de Home Screen (9).

8) Recover Password Screen: In this screen the user will enter the register email to retrieve account.

9) Home Screen: There are three options to choose from: Edit Profile (10), Summary Reports History (12), and Log Out (14)

10) Edit Profile: User will be able to edit the account information or update the medical history.

11) Pair Device: Every time the user updates account it will have to be paired with device.

12) Summary Reports History: User will be able to choose from a list of all his summary reports.

13) Summary Report: User will be able to view the chosen Summary Report from the Summary Report History Screen (12).

14) Log Out Screen: Logs out of the application.

\section{B. Paramedics application}

Fig. 8 presents the paramedics' application workflow. Each step works as follows:

1) Login Screen: user enters username and password. If user enters invalid credentials, will be directed to the Invalid Credentials Screen (2). Otherwise, the user will be directed to the Home Menu Screen (4).

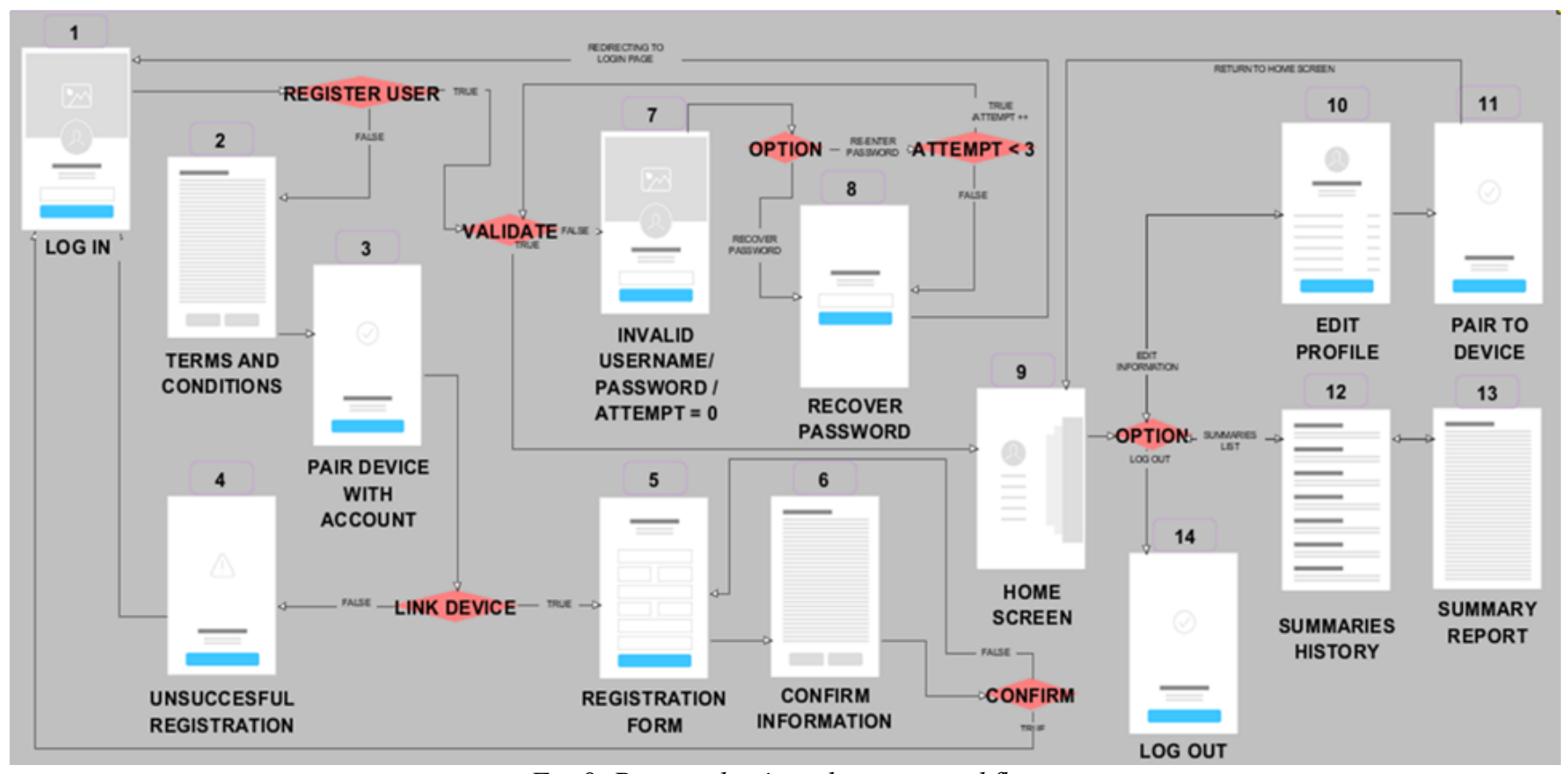

Fig 8. Paramedics' application workflow 
2) Invalid Credentials: If the user validation is unsuccessful in Login Screen (1) it will go to this page. The user will have 3 try attempts. After three unsuccessful attempts, user's account will be blocked. It will appear the Account Blocked Screen (3).

3) Account Blocked: This screen will appear when the user makes three invalid login attempts. It will have an "OK" button that will redirect the user to the Login Page (1). To maintain a certain level of security, user will have to contact support to unblock account.

4) Home Menu Screen: There are four options to choose from: Create Encounter, Saved Encounters (7), Submitted Encounters (9), and Log Out (11).

5) Upload from Patient's Device: If user chooses to create a new encounter, this screen will appear. Once the patient's information will be able to open a new Encounter form (6).

6) Encounter form screen: The user will fill out this encounter form while patient is receiving treatment. User may have the option to either save encounter to finish later or submit it.

7) Encounter form screen: The user will fill out this encounter form while patient is receiving treatment. User may have the option to either save encounter to finish later or submit it.

8) List of Saved Encounters: User will be able to choose from a list of all his saved encounters.

9) Selected Encounter: User will be able to edit the chosen encounter from the List of Saved Encounter Screen (7). It can be saved again or submitted. In case of being submitted, it will be added in the Summary Report History Screen (9).

10) Summary Report History: User will be able to choose from a list of all the summary reports that was submitted by him.

11) Selected Report: User will be able to view the chosen Summary Report from the Summary Report History Screen (9).

12) Log Out Screen: Logs out of the application.

\section{SUMMARY}

This work presented a system called CARElink, which was designed to allow a paramedic to access a patient's medical history in real time during a medical emergency. Many findings were established across the design development of this project. Parting from the problem definition, and steadily beginning to broad the scope of the project. Being able to validate such means and realizing a vast research not only to validate the problem, but also in finding an alternative to solve it. Our research indicated that indeed the response time does matter since the mortality odds increase by the minute.
Throughout this system design development, we acquired experience validating our problem and solution by research and interviews. We identified constraints, security issues, information management issues, and so more. Other observations may include the synchronization between the portable device database and the global database, which was solved by designing the local database from the portable device as a subset of the global database. During this time, we dealt with the portable device design development. It had to accommodate to the ideal setting of any emergency, that didn't depend on telecommunications, have low energy consumption and data could be accessible at any given time.

After a vast analysis and considering the energy savings for the portable device, we selected an alternative where an USB is used in conjunction with a RFID. The reason for this is because the USB does not consume power when it is turned off and, is cost effective. The RFID is exclusively for the paramedic to track the patient's portable device during an emergency, so it could avoid wasting time during the evaluation and treatment. This alternative is very favorable since the stored information will always be available without the need to be charging the device.

During this past 6 weeks we were able to start implementing the graphical user interface (GUI) on both applications. We also completed both scripts for our local and global databases. After we determined the type of device to be used in order to store the patient's information, we focused on achieving the data transferring from the patient's application to the portable device. Once accomplished, we continued to work with the data transferring from the portable device to the paramedic's application. As result, the aforementioned objectives were satisfactorily fulfilled.

\section{REFERENCES}

[1] NENA: The 9-1-1 Association "9-1-1 Statistics," https://www.nena.org/ [2] RapidSOS: "Outcomes: Quantifying the Impact of Emergency Response Times". www.rapidSOS.com

[3] Ian E. Blanchard, Christopher J. Doig, Brent E. Hagel, Andrew R. Anton, David A. Zygun, John B. Kortbeek, D. Gregory Powell, Tyler S. Williamson, Gordon H. Fick \& Grant D. Innes (2012) Emergency Medical Services Response Time and Mortality in an Urban Setting, Prehospital Emergency Care, 16:1, 142-151

[4] Wilde, E. T. (2013), DO EMERGENCY MEDICAL SYSTEM RESPONSE TIMES MATTER FOR HEALTH OUTCOMES? Health Econ., 22: 790-806.

[5] Pons, P. T., Haukoos, J. S., Bludworth, W, Cribley, T, Pons, K. A. and Markovchick, V. J. (2005), Paramedic Response Time: Does It Affect Patient Survival? Academic Emergency Medicine, 12: 594-600.

[6] Blackwell and Kaufman, "Response Time Effectiveness: Comparison of Response Time and Survival in an Urban Emergency Medical Services System", ACADEMIC EMERGENCY MEDICINE, Vol. 9, No. 4, pp. 292, April 2002. 